\title{
Direct-Write Printing of Josephson Junctions in a Scanning Electron Microscope
}

Tycho J. Blom, Thomas W. Mechielsen, Remko Fermin, Marcel B. S. Hesselberth, Jan Aarts, and Kaveh Lahabi*

Cite This: ACS Nano 2021, 15, 322-329

Read Online

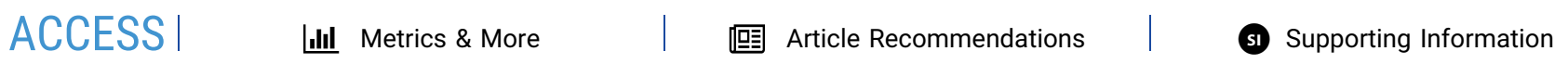
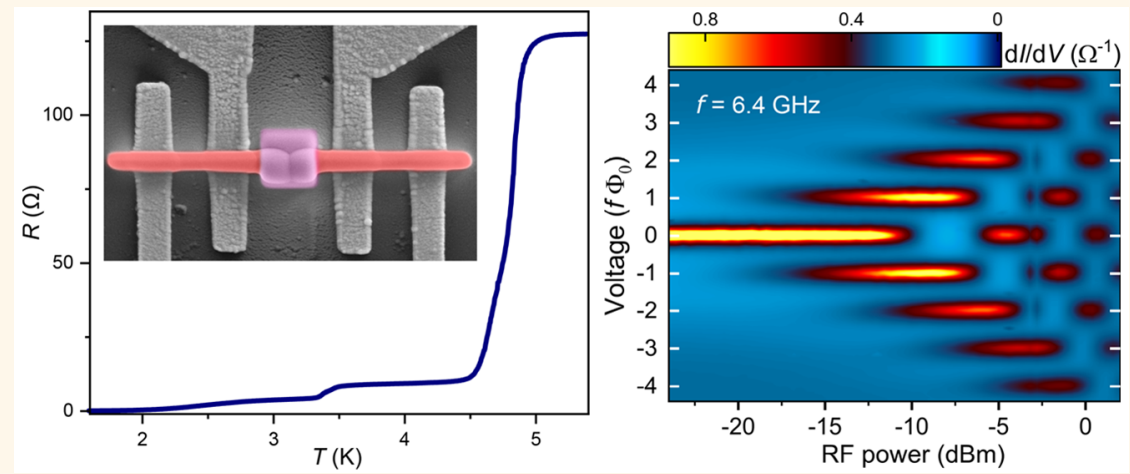

ABSTRACT: Josephson junctions are the building blocks of superconducting electronics, with well-established applications in precision metrology and quantum computing. Fabricating a Josephson junction has been a resourceintensive and multistep procedure, involving lithography and wet-processing, which are not compatible with many applications. Here, we introduce a fully additive direct-write approach, where a scanning electron microscope can print substrate-conformal Josephson devices in a matter of minutes, requiring no additional processing. The junctions are made entirely by electron-beam-induced deposition (EBID) of tungsten carbide. We utilize EBIDtunable material properties to write, in one go, full proximity junctions with superconducting electrodes and metallic weak links and tailor their Josephson coupling. The Josephson behavior of these junctions is established and characterized by their microwave-induced Shapiro response and field-dependent transport. Our efforts provide a versatile and nondestructive alternative to conventional nanofabrication and can be expanded to print threedimensional superconducting sensor arrays and quantum networks.

KEYWORDS: Josephson junction, direct-write fabrication, focused electron-beam-induced deposition, superconductivity, tungsten carbide, superconducting transport

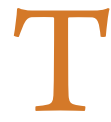

he Josephson effect arises when macroscopic quantum states are separated by a medium which allows their wave functions to partially overlap. The phenomenon takes place in Josephson junctions, where the macroscopic wave functions of superconducting electrodes are coupled via some form of a barrier. Unlike conventional electronic devices, where electrical current is driven by a potential difference (i.e., voltage), charge transport in a Josephson junction is governed by the difference in the quantum mechanical phase of superconducting electrodes. The current-phase relation enables Josephson junctions to combine the efficiency of dissipation-less transport with the precision of quantum interferometry. Today, Josephson junctions are an indispensable ingredient in a variety of fields, from highly sensitive detectors, used for imaging and signal processing, to quantum circuitry and superconducting computing.

So far, the fabrication of Josephson devices has been a multistep process. This is typically a combination of thin-film deposition and additional structuring/patterning step(s), which can involve lithographic processes (e.g., spin-coating of resist and etching) or exposure to focused ion beams (FIBs). ${ }^{1}$ These procedures are not always compatible with potential

Received: $\quad$ May 1, 2020

Accepted: November 11, 2020

Published: November 24, 2020 

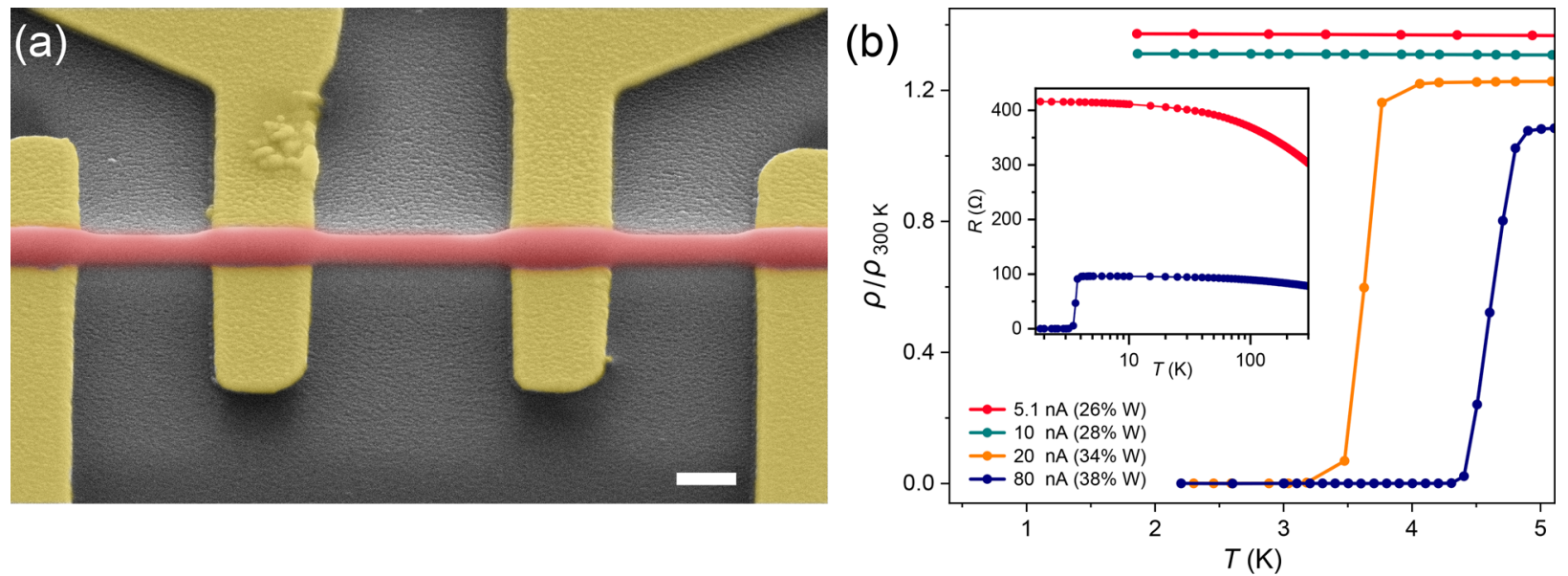

Figure 1. (a) False-color scanning electron micrograph of a W-C wire (red), contacted with gold electrodes (yellow). The scale bar corresponds to $500 \mathrm{~nm}$. (b) Normalized resistivity of $\mathrm{W}-\mathrm{C}$ wires deposited by beam currents ranging between 5.1 and $80 \mathrm{nA}$. Each wire is deposited with $25 \mathrm{~ms}$ dwell time and $60 \%$ beam overlap. Resistance is measured using a $10 \mu \mathrm{A}$ dc bias. Legends show the $\mathrm{W}$ concentrations (atomic percentages) estimated from EDX analysis. The inset compares the temperature-dependent resistance of the wires made by 5.1 and 80 nA beams.

applications of superconducting devices, for instance, in cases where a fragile substrate or a nonplanar surface cannot be resist-coated or when exposure to ion beams or immersion in liquids is detrimental to the system. There is currently an absence of nondestructive direct-write methods for fabricating Josephson devices.

Here, we present a one-step additive technique to "print" Josephson junctions using electron-beam-induced deposition (EBID), i.e., by scanning an electron beam to locally dissociate precursor molecules, which are subsequently adsorbed on the surface. The entire fabrication procedure takes place in a scanning electron microscope (SEM) with a gas injection system, which also allows for direct quality control and device repair. A complete Josephson junction can be printed in a matter of minutes. Except for the contacts to the outside electronics, the EBID Josephson junctions do not require thinfilm deposition or additional processing (e.g., annealing, exposure to chemicals, or ion beams). This provides a noninvasive means to add high-sensitivity detectors, such as superconducting quantum interference devices (SQUIDs) for magnetometry at the desired location of an existing structure.

The Josephson junctions are made of a tungsten carbide (W-C) compound, formed by the dissociation of tungsten hexacarbonyl $\mathrm{W}(\mathrm{CO})_{6}$ molecules. Whereas the superconducting transition temperature $\left(T_{\mathrm{c}}\right)$ of pure crystalline tungsten is only $0.011 \mathrm{~K}, 2$ disordered and amorphous tungsten alloys with $\mathrm{Si}, \mathrm{C}$, and $\mathrm{Ge}$ can have a $T_{\mathrm{c}}$ around $6 \mathrm{~K}^{3}$ The precursor $\mathrm{W}(\mathrm{CO})_{6}$ has been widely implemented for ion-beam-induced deposition (IBID), usually with a focused $\mathrm{Ga}^{+}$or $\mathrm{He}^{+}$beam, to fabricate superconducting structures. ${ }^{4-11}$ Exposure to ion beams, however, is unavoidably accompanied by ion implantation and beam-induced disorder, which are detrimental to pristine systems (e.g., graphene). Furthermore, the inevitable sputtering associated with ion bombardment introduces additional constraints on the capacity of IBID for three-dimensional (3D) printing. EBID does not suffer such drawbacks; the absence of ion implantation and sputtering makes this technique far less destructive and more compatible with the principles of $3 \mathrm{D}$ printing.

Despite the clear benefits, however, there has been little success in producing superconducting structures with EBID. In the case of $\mathrm{W}-\mathrm{C}$, most studies report poor electrical conductivity and no sign of a superconducting phase in structures made with EBID. ${ }^{5,12,13}$ There is currently only one report of superconducting $\mathrm{W}-\mathrm{C}$ wires made with EBID, with the highest $T_{\mathrm{c}}$ reaching $2 \mathrm{~K},{ }^{14}$ which is still considerably lower than the values obtained with IBID. The challenge in producing superconducting structures with EBID has not been specific to $\mathrm{W}(\mathrm{CO})_{6}$. Even when including other precursors, at present, there are merely three reports of superconducting EBID wires. ${ }^{14-16}$ The lack of superconducting EBID structures has also led to a long-standing debate over the possible role of $\mathrm{Ga}$ atoms in the emergence of superconductivity in the $\mathrm{W}$-based materials deposited with IBID. ${ }^{17}$ Here, we demonstrate that superconducting $\mathrm{W}-\mathrm{C}$ with enhanced $T_{c}$ can also be produced without $\mathrm{Ga}$; we use EBID to write $\mathrm{W}-\mathrm{C}$ wires with $T_{\mathrm{c}}$ reaching up to $5.7 \mathrm{~K}$. We utilize the wires to inject superconducting correlations in metallic $\mathrm{W}-\mathrm{C}$ weak links to create direct-write proximity Josephson junctions. We show that EBID parameters, such as beam current and scanning speed, can be implemented to write the superconducting electrodes and the metallic weak link in one go and to tune the critical current $I_{\mathrm{c}}$ and normal-state resistivity $\rho_{\mathrm{N}}$ of the junctions.

\section{RESULTS AND DISCUSSION}

Superconducting Electrodes. We begin with the transport characteristics of $\mathrm{W}-\mathrm{C}$ wires, which serve as the superconducting electrodes in our junctions. The wires are deposited on a $\mathrm{Si}_{3} \mathrm{~N}_{4}$-coated silicon substrate with lithographically patterned gold electrodes for transport measurements (see Figure 1a). Each wire is written by scanning a 10 $\mathrm{keV}$ electron beam in a straight line. Efficient EBID requires a high local density of electrons, which can dissociate the organometallic precursor molecules. It has been reported that higher beam currents can enhance the metal content by increasing the number of available electrons per precursor molecule. ${ }^{18}$ This is also the case here, as demonstrated in Figure $1 b$, which compares the temperature-dependent resistivity of wires deposited with different beam currents, normalized by the resistance of the wire at $T=300 \mathrm{~K}$ (a detailed overview of the EBID parameters used for each 
structure is provided in Supporting Information). By increasing the beam current above $5 \mathrm{nA}$ - a regime which was typically not probed in previous studies-we observe a substantial enhancement of the metallicity and the emergence of superconductivity. Energy-dispersive X-ray spectroscopy (EDX) shows that the W concentration increases from $26 \%$ (atomic percentage) at $5.1 \mathrm{nA}$ beam current to $38 \%$ at $80 \mathrm{nA}$ (see the legend in Figure 1b). Combining the EDX results with the temperature-dependent transport measurements reveals a direct correlation between metal content, the behavior of the normalized resistivity, and the occurrence of superconductivity.

We use $20 \mathrm{nA}$ as the standard beam current for fabricating our junctions. This corresponds to a beam spot diameter of around $60 \mathrm{~nm}$ in our instrument. For this beam current, the optimal values of dwell time and pitch (step size) are found to be $25 \mathrm{~ms}$ and around $1 \mathrm{~nm}$, respectively (note that the optimal values differ from those used for writing the wires of Figure 1). These parameters result in wires with a typical $T_{\mathrm{c}}$ of $4.7 \mathrm{~K}$ (see Figure $2 \mathrm{~b}$ ) and a room temperature resistivity of $\rho \approx 270 \mu \Omega$ $\mathrm{cm}\left(\rho_{(300 \mathrm{~K})} / \rho_{(10 \mathrm{~K})} \approx 0.85\right)$. Increasing the beam current to 80 $\mathrm{nA}$ can raise the $T_{\mathrm{c}}$ even further to $5.7 \mathrm{~K}$ (see Supporting Information). Such high currents, however, can lead to electrostatic charging effects and larger beam spots, which limit our control over device fabrication. The bottom line here is that EBID can provide superconducting $\mathrm{W}-\mathrm{C}$ with a $T_{\mathrm{c}}$ that is comparable to those written by IBID using $\mathrm{Ga}^{+}$ions. ${ }^{4-9}$

Weak Link Material. A Josephson junction requires a weak link that segregates the superconducting condensates of the electrodes, while also coupling their wave functions to yield a single-valued current-phase relation. Naturally, the superconducting order parameter needs to be locally suppressed at the weak link. We achieve this by connecting superconducting $\mathrm{W}-\mathrm{C}$ wires via a separate $\mathrm{W}-\mathrm{C}$ segment with lower metallicity, where superconductivity is suppressed. Above, we demonstrated that beam current has a substantial influence over the electrical characteristics of the $\mathrm{W}-\mathrm{C}$ and can be tuned to obtain both superconducting and normal metal components with which to fabricate junctions. However, writing the weak link with a different beam current than the electrodes would not be practical due to the additional refocusing necessary when switching the aperture of a SEM. A more effective approach is to utilize the dwell time, i.e., the time the electron beam spends per pixel. Longer dwell times are known to enhance the metallicity by increasing the number of electron scattering events per precursor molecule, which leads to a more efficient carbonyl (CO) desorption. ${ }^{18,19}$ We examine this using rectangular $\mathrm{W}-\mathrm{C}$ ribbons, which serve as prototypes for the weak link materials used in the final EBID junctions. The ribbons are deposited by raster scanning a $20 \mathrm{nA}$ beam in steps of $23 \mathrm{~nm}$ over a $200 \mathrm{~nm}$ wide rectangle. Depending on the dwell time, the number of raster scans used for each ribbon is adjusted to maintain the total applied dose constant. Figure $2 \mathrm{a}$ shows the resistivity of the $\mathrm{W}-\mathrm{C}$ ribbons at $4 \mathrm{~K}\left(\rho_{4 \mathrm{~K}}\right)$, plotted as a function of dwell time. The error bars correspond to the uncertainty involved in estimating the cross-sectional area of the ribbons.

We observe a significant reduction of resistivity by increasing the dwell time to $10 \mathrm{~ms}$, where it appears to approach a lower limit. The results indicate that there is a range of dwell times which can be used for tuning weak link conductivity. In Figure $2 \mathrm{~b}$, we compare the transport behavior of a high-quality $\mathrm{W}-\mathrm{C}$ wire, written using $25 \mathrm{~ms}$ dwell time (line scan in $1 \mathrm{~nm}$ steps), together with a $\mathrm{W}-\mathrm{C}$ ribbon of the same length, deposited
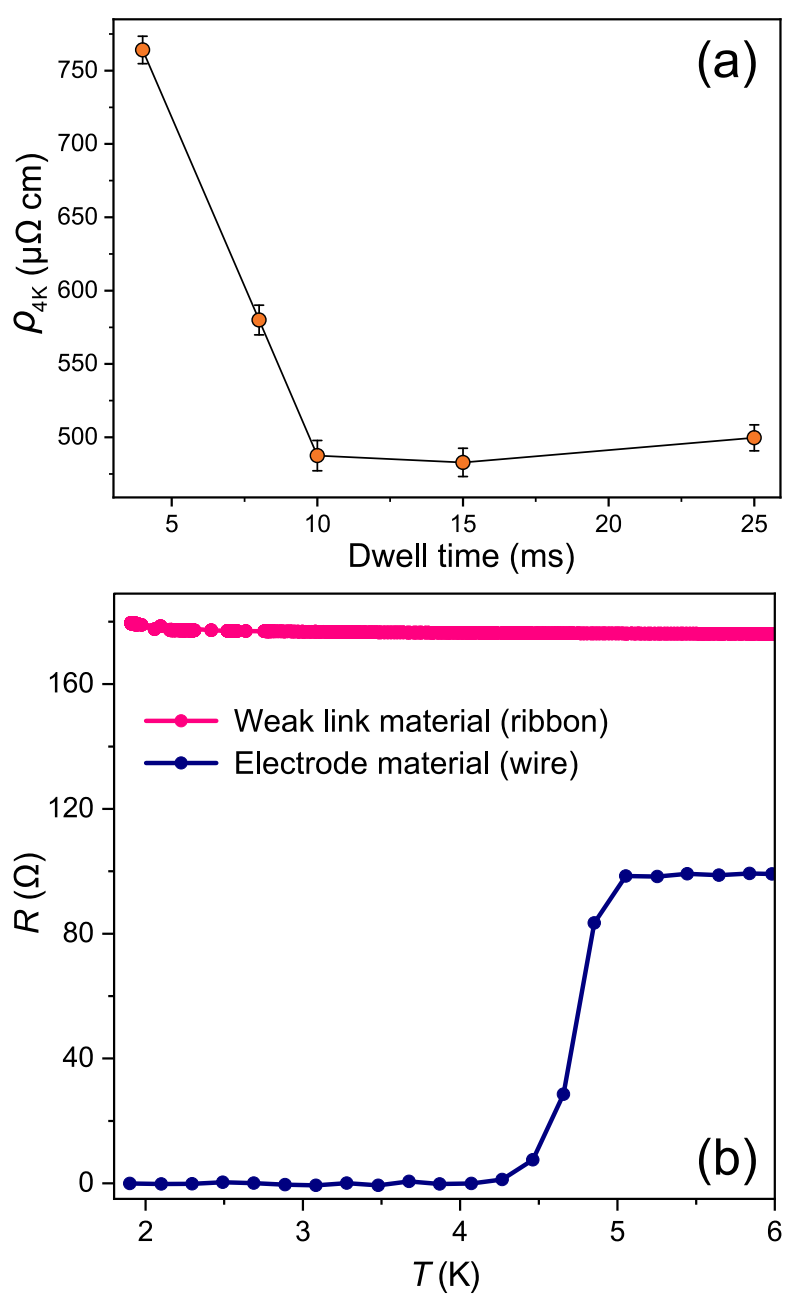

Figure 2. (a) Resistivity of $\mathrm{W}-\mathrm{C}$ ribbons (prototypes for weak link) at $4 \mathrm{~K}$ as a function of EBID dwell time. (b) Temperaturedependent resistance of a superconducting $\mathrm{W}-\mathrm{C}$ electrode wire, made using optimal parameters (blue), and a non-superconducting $\mathrm{W}-\mathrm{C}$ ribbon (pink), both measured using a $10 \mu \mathrm{A}$ bias. The structures are deposited using a $20 \mathrm{nA}$ beam current and are of similar dimensions. The wire (written in line scan mode with 25 $\mathrm{ms}$ dwell time) and the ribbon (written by raster scans with $4 \mathrm{~ms}$ dwell time) represent the transport characteristics of the superconducting electrodes and the non-superconducting proximity weak links used in the final junctions, respectively.

with $4 \mathrm{~ms}$ dwell time (raster scan with $23 \mathrm{~nm}$ step size). The former is used in the superconducting electrodes of our EBID junctions, whereas the latter is representative of the weak link material that couples the electrodes together.

Josephson Junctions. Figure 3 a shows a typical EBID Josephson junction. The junctions are prepared by first depositing the superconducting electrodes, which are around $160 \mathrm{~nm}$ apart (Figure 3a, inset). The electrode wires are subsequently connected by a weak link, a $0.5 \mu \mathrm{m}$ sized $\mathrm{W}-\mathrm{C}$ square, deposited with a shorter dwell time, using a raster scan. It is worth mentioning that we found no electrical contribution from the delocalized (halo) deposits, which are known to form around EBID structures. Without the weak link material to connect the electrodes, no current could be measured across the $160 \mathrm{~nm}$ gap. Figure $3 \mathrm{~b}-\mathrm{d}$ shows the basic transport properties for three different Josephson junctions made in this way. JJ1 and JJ2 are printed using the same parameters for the 
(a)

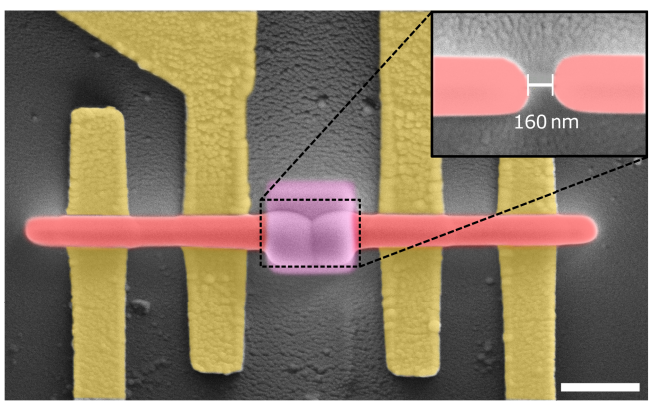

(b)

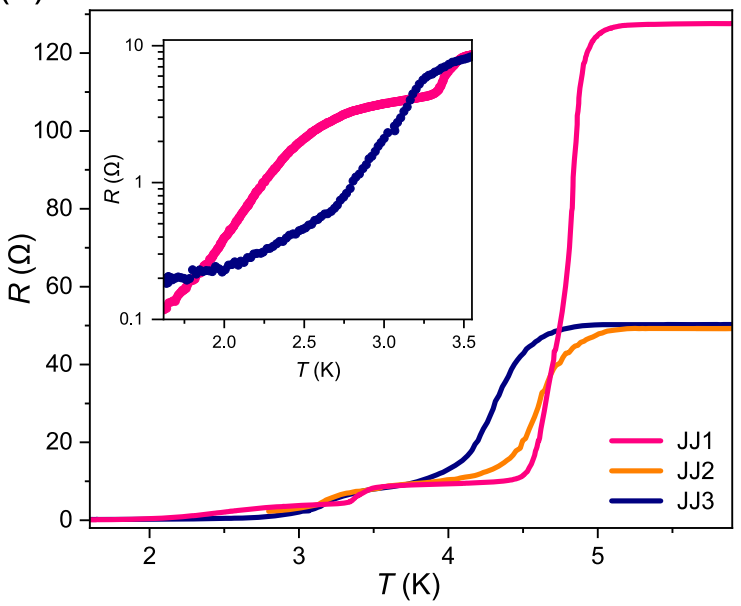

(c)

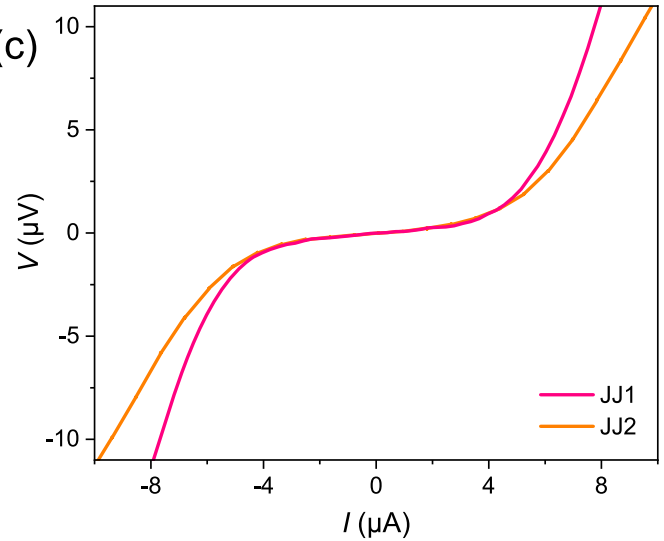

(d)

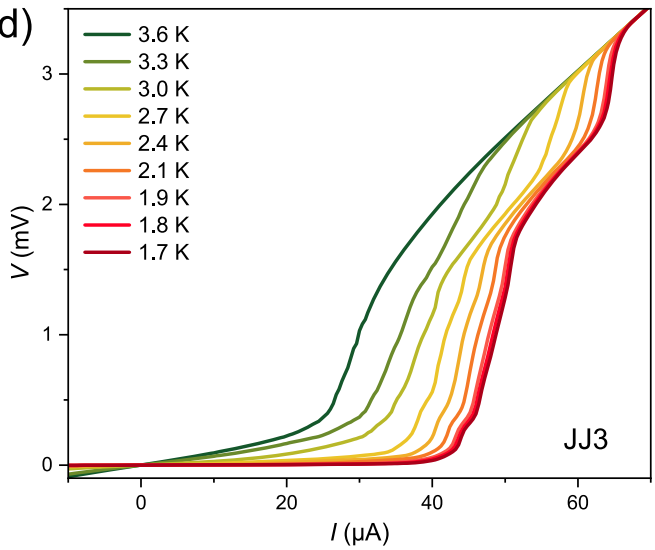

Figure 3. (a) False-color micrograph of an EBID Josephson junction. A W-C weak link (purple) connects two superconducting W-C electrodes (red), printed on gold contacts (yellow). Scale bar represents $1 \mu \mathrm{m}$. Inset image, taken prior to the deposition of the weak link material, shows the $160 \mathrm{~nm}$ gap that separates the superconducting electrodes and represents the effective length of the weak link. (b) Temperature-dependent resistance of three EBID junctions, measured with a $10 \mu \mathrm{A}$ bias current. Inset shows the proximity-induced transition of $\mathrm{JJ} 1$ and $\mathrm{JJ} 3$ weak links, plotted on a logarithmic scale. (c) $I-V$ characteristics of $\mathrm{JJ} 1$ and $\mathrm{JJ} 2$ weak links (both made with 4 ms dwell time), taken at $1.5 \mathrm{~K}$. (d) $I-V$ characteristics measured at different temperatures for JJ3 (weak link made using $10 \mathrm{~ms}$ dwell time).

weak link, with a $4 \mathrm{~ms}$ dwell time. This dwell time is increased to $10 \mathrm{~ms}$ for $\mathrm{JJ} 3$ in order to enhance its metallicity. Figure $3 \mathrm{~b}$ shows the temperature-dependent resistance of the three junctions, measured with a $10 \mu \mathrm{A}$ bias. It should be noted that JJ1 is deposited on a different substrate, where the distance between the gold voltage contacts is around three times larger, resulting in a higher normal-state resistance. The $R(T)$ of each junction exhibits two main transitions: one at higher temperatures (typically around $4.5 \mathrm{~K}$ ), representing the superconducting transition of the electrodes, and a broader one at noticeably lower temperatures (see the inset), which corresponds to the emergence of proximity-induced correlations in the weak link. Below the second transition, EBID junctions develop a clear $I_{\mathcal{C}}$ as demonstrated by the currentvoltage $(I-V)$ measurements in Figure 3c,d. Furthermore, we find no hysteresis in the $I-V$ characteristics.

Figure $3 \mathrm{c}$ shows that applying the same scanning parameters to deposit the weak links of JJ1 and JJ2 results in a matching $I_{\mathrm{c}}$ for both junctions. This is despite the fact that the junctions are fabricated on separate substrates and on different days. For junctions made with $4 \mathrm{~ms}$ dwell time, the typical $I_{\mathrm{c}} R_{\mathrm{N}}$ product is between 10 and $14 \mu \mathrm{V}$. Here, $R_{\mathrm{N}}$ corresponds to the normalstate resistance of the weak link and is determined by the value of $\mathrm{d} V / \mathrm{d} I$ for $I>I_{\mathrm{c}}$ at $T \approx 1.5 \mathrm{~K}$ (when the weak link is fully proximized). The weak link of JJ3 was deposited with a higher dwell time (10 ms) compared to JJ1 and JJ2 (4 ms). Hence, due to the enhanced metallicity of its weak link, JJ3 yields a substantially higher $I_{\mathrm{c}}$ (see Figure $3 \mathrm{~d}$ ), corresponding to $I_{\mathrm{c}} R_{\mathrm{N}}$ $\approx 50 \mu \mathrm{V}$.

It should be noted that, at $T=1.5 \mathrm{~K}$, the base temperature of our cryostat, the junctions exhibit some finite resistance. For $\mathrm{JJ} 3$, this is $0.12 \Omega$, which is $10 \%$ of the normal-state resistance of the weak link, whereas JJ1 and JJ2 both have a resistance of around $0.11 \Omega$, which corresponds to $3-6 \%$ of their respective $R_{\mathrm{N}}$. The residual resistance can be seen as the slight slope in the $I-V$ characteristics for $I<I_{c}$ (see Figure 3c). This finite resistance may vanish by lowering the temperature or it may maintain a finite value due to some form of a phase slip process. While its exact origin is not clear at this stage, as demonstrated below, the resistance is not caused by a lack of coherent Josephson transport.

To unambiguously establish the Josephson transport in our EBID devices, we measured their Shapiro response to a radio frequency (RF) excitation. When external RF radiation couples to the junction dynamics by driving an additional RF supercurrent, it leads to the emergence of Shapiro steps in the $I-V$ characteristics. These are discrete voltage steps that appear at $V=n h f / 2 e=n f \Phi_{0}$, where $f$ is the microwave frequency, $n$ is an integer, and $\Phi_{0}$ is the superconducting magnetic flux quantum. The Shapiro response of our junctions was verified by irradiating them with microwave frequencies from a nearby antenna (an open-ended coax, positioned a few millimeters above the sample) while measuring their $I-V$ characteristics using a dc current bias. Figure 4 provides clear 


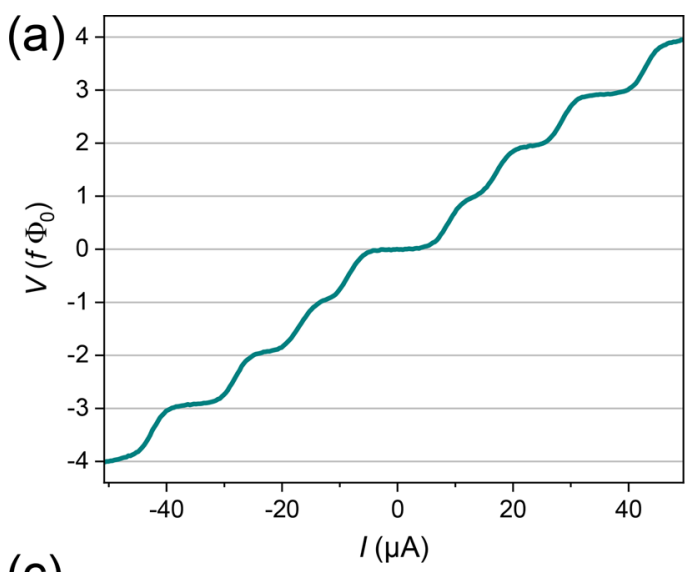

(c)

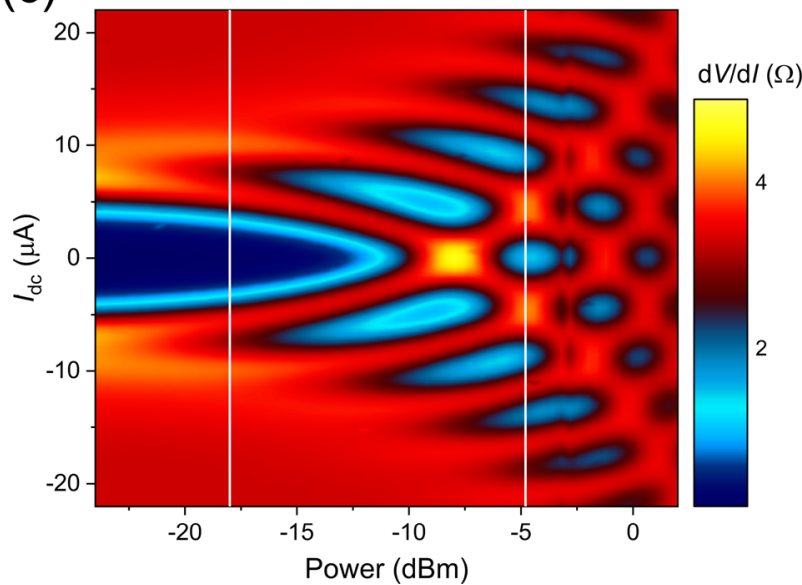

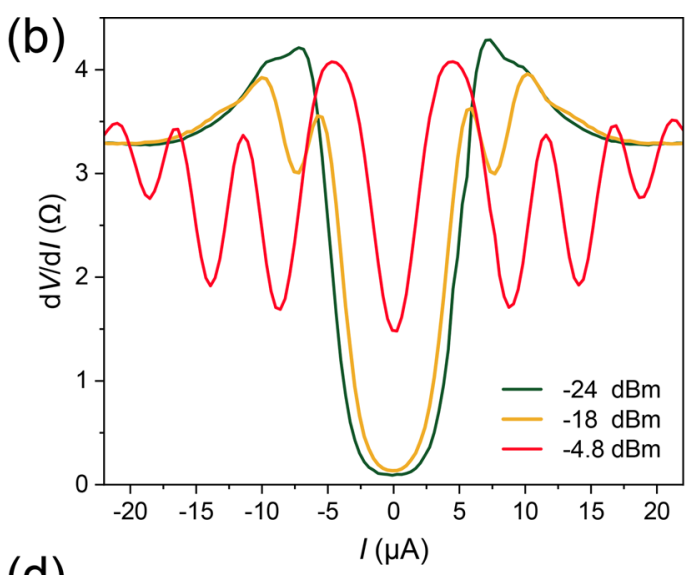

(d)

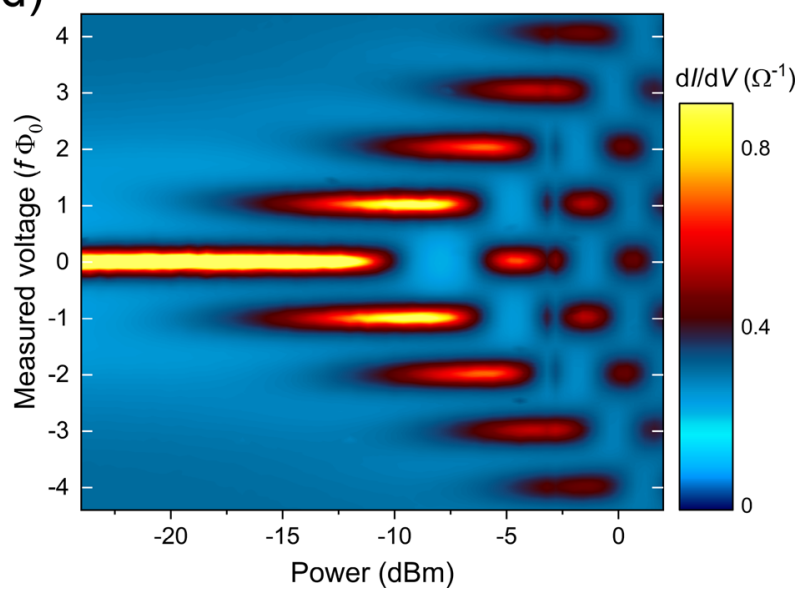

Figure 4. Shapiro response of junctions $\mathrm{JJ} 1$ and $\mathrm{JJ} 3$ to microwave irradiation of $f=6.4 \mathrm{GHz}$ frequency. (a) $I-V$ characteristic of $\mathrm{JJ} 3$, measured at $1.5 \mathrm{~K}$. To emphasize the Shapiro steps, the voltage scale is normalized in units of $f \Phi_{0}$. The linear background, caused by the residual resistance of the device, has been subtracted from the $I-V$. (b) Differential resistance $(\mathrm{d} V / \mathrm{d} I)$ of $\mathrm{JJ} 1$ at $1.64 \mathrm{~K}$, measured for different RF powers. Shapiro steps manifest themselves as the "valleys" in the $d V / d I$, which emerge by increasing the power. (c,d) Evolution of the Shapiro response of JJ1 with RF power, shown as colormaps of $d V / d I(c)$ and differential conductance $d I / d V(d)$. White lines in (c) correspond to the measurements in (b). To demonstrate the quantized Shapiro response, the voltage in (d) is scaled in units of $f \Phi_{0}$.

examples of the Shapiro response measured for 10 and $4 \mathrm{~ms}$ EBID junctions (JJ3 and JJ1, respectively). Figure 4a shows the Shapiro steps in the $I-V$ curve of JJ3. In order to show the $f \Phi_{0}$ quantization of the steps in Figure $4 \mathrm{a}$, the linear background, caused by the residual resistance at $1.5 \mathrm{~K}$, has been subtracted from the $I-V$. The Shapiro response of JJ1 is shown by the differential resistance $(\mathrm{d} V / \mathrm{d} I)$ curves in Figure $4 \mathrm{~b}$. For each junction, the steps occur at exactly integer multiples of $f \Phi_{0}$. The effect was reproduced for various microwave frequencies.

We also explored the evolution of Shapiro steps with RF power. This was carried out by mapping the $\mathrm{d} V / \mathrm{d} I$ as a function of RF power and dc current (swept from negative to positive bias). The results for JJ1 are presented as colormaps in Figure $4 c, d$ in the form of differential resistance and differential conductance $(\mathrm{d} I / \mathrm{d} V)$, respectively. To demonstrate the quantization of the Shapiro response more clearly, the measured voltage in Figure $4 \mathrm{~d}$ is normalized in units of $f \Phi_{0}$. The power-dependent Shapiro response of JJ3 is included in the Supporting Information.

In addition to the Shapiro response, we use magnetic fielddependent measurements as an independent approach to verify the Josephson transport in our devices. The field-dependent critical current $I_{\mathrm{c}}(B)$ of a single Josephson junction is often associated with the well-known Fraunhofer diffraction pattern, in which $I_{c}$ goes to zero when the magnetic flux applied to the junction is an integer multiple of $\Phi_{0}$. However, depending on their exact shape, dimensions, and material characteristics, Josephson junctions can exhibit widely different $I_{c}(B)$ patterns. The elastic mean free path of $\mathrm{W}-\mathrm{C}(l \approx 0.3 \mathrm{~nm})^{20,21}$ is much shorter than the superconducting coherence length $(\xi \approx 6$ $\mathrm{nm}),{ }^{22}$ which, in turn, is smaller than the length of the junction $(L \approx 160 \mathrm{~nm})$, making the EBID junctions long and highly diffusive. It has been demonstrated that for long and diffusive junctions with narrow contacts, where the length and width of the weak link are comparable to each other, and also smaller than the penetration depth $\left(\lambda=1.05 \times 10^{-3} \sqrt{\rho_{\mathrm{N}} / T_{\mathrm{c}}} \approx 830\right.$ $\mathrm{nm}),{ }^{23} I_{\mathrm{c}}(B)$ follows a Gaussian-like decay, given by

$$
I_{c}(B)=I_{c}(0)\left|e^{-\left(\frac{B S}{\Phi_{0}}\right)^{2} /\left(2 \sigma^{2}\right)}\right|
$$

where $I_{c}(0)$ is the zero-field $I_{\mathcal{O}} S$ is the effective area of the weak link (in this case $6 \times 10^{-14} \mathrm{~m}^{2}$ ), and the Gaussian coefficient $\sigma$ is determined by the diffusive trajectories of Andreev pairs. As described previously, ${ }^{24,25}$ the value of $\sigma$ varies according to the exact geometry of the junction. As our EBID junctions are equivalent in geometry, we expect their field-dependent behavior to be described by the same $\sigma$ and $S$ values. We examine this by comparing the $I_{\mathrm{c}}(B) / I_{\mathrm{c}}(0)$ of $\mathrm{JJ} 1$ and JJ3. These two junctions are equivalent in terms of 

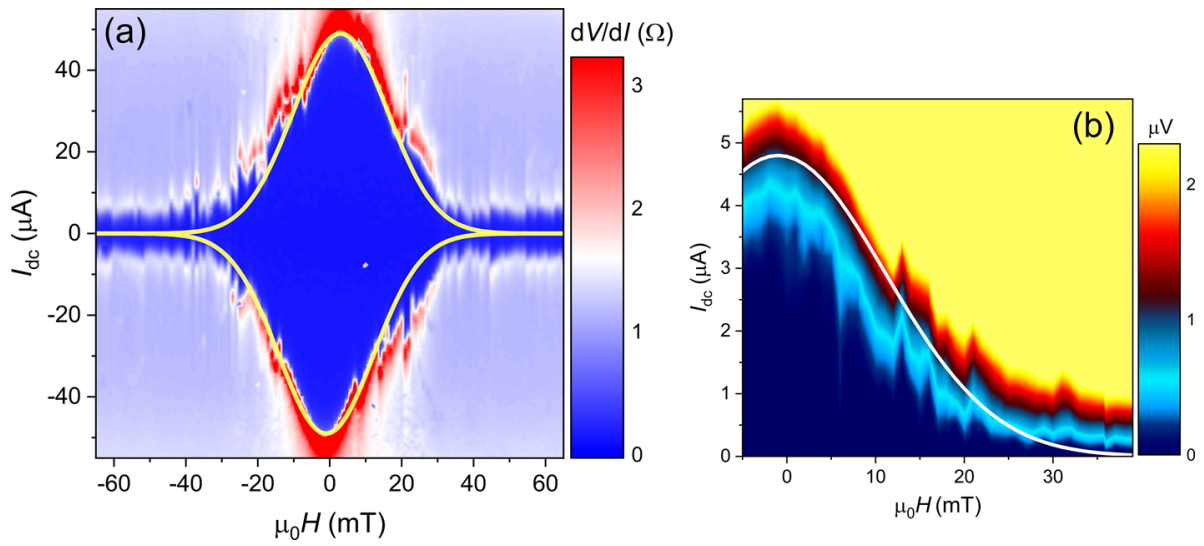

Figure 5. (a) Field-dependent $\mathrm{dV} / \mathrm{d} I$ of $\mathrm{JJ} 3$ for positive and negative current bias, measured at $1.5 \mathrm{~K}$ by sweeping the out-of-plane magnetic field (from negative to positive) in $1 \mathrm{mT}$ steps. (b) Field-dependent response of JJ1, obtained by $I-V$ scans taken while sweeping the field $(40$ to $-5 \mathrm{mT})$. The results in (a) and (b) are both fitted with the same Gaussian expression, as described by eq 1 .

geometry but vary in $I_{\mathcal{c}}$, due to the different dwell times used for depositing their weak links. In Figure 5, we plot the $\mathrm{d} V / \mathrm{d} I$ of JJ3 together with the $I-V$ characteristics of $J J 1$ as a function of applied out-of-plane magnetic field. Despite the considerable difference in their zero-field $I_{c}$, the supercurrent vanishes around $30 \mathrm{mT}$ in both junctions. More specifically, each junction follows the same $I_{c}(B) / I_{c}(0)$ pattern, as described by eq 1 , and can be fitted with $\sigma=0.4$ in both cases (see the solid lines in Figure 5). Here, $\sigma$ is the only free parameter used in our fits, and its magnitude is consistent with previous reports on diffusive junctions of similar configuration. ${ }^{24,25}$ It follows from the analysis above that the critical current of the EBID junctions can be tuned over a wide range, independently of device dimensions and geometry. This is particularly relevant for SQUIDs and flux qubit circuits, where $I_{c}$ matching is an ongoing challenge.

Lastly, we point out that the Josephson effect only occurs in structures with a well-defined weak link that can introducing a stable boundary between the condensates of the electrodes. Individual $\mathrm{W}-\mathrm{C}$ wires exhibit no Shapiro response or a Gaussian-like $I_{\mathrm{c}}(B)$ pattern; they maintain a finite $I_{\mathcal{c}}$ which gradually disappears as the field approaches the upper critical field of $\mathrm{W}-\mathrm{C}, \mathrm{H}_{\mathrm{c} 2} \approx 4 \mathrm{~T}$. An inhomogeneous wire which still acts as a single condensate cannot yield a single-valued currentphase relation to support Josephson transport. An example of such a system is discussed in the Supporting Information.

\section{CONCLUSION AND OUTLOOK}

To summarize, we apply EBID to develop a fully additive direct-write approach to fabricating full proximity Josephson junctions inside an SEM, where both superconducting and normal metal components can be printed by regulating the parameters of the scanning electron beam. This principle is extended to provide a reliable means for tuning the critical current of the junctions. We also demonstrate that EBID can produce superconducting $\mathrm{W}-\mathrm{C}$, with $T_{\mathrm{c}}$ reaching as high as $5.7 \mathrm{~K}$, which so far had only been reported for $\mathrm{W}-\mathrm{C}$ structures made with IBID.

Given the widespread availability of electron microscopes, the EBID approach presented here provides an accessible and relatively inexpensive means to fabricate Josephson devices such as SQUIDs and single-photon detectors. ${ }^{26}$ The ability to directly print a Josephson junction on a given surface, without the use of energetic ions or wet processing, expands the current applications of superconducting electronics. This is particularly advantageous when dealing with nonplanar surfaces, mechanically fragile substrates (e.g., membranes, free-standing beams, and cantilevers) and systems that are vulnerable to chemical processing. Even more enticing is the capacity of EBID for 3D nanoprinting. With the advent of hybrid Monte Carlocontinuum simulations and computer-aided design programs, pioneered by Fowlkes et al. ${ }^{27}$ and Huth and Keller, ${ }^{28}$ the field of 3D EBID has received a renewed boost in recent years. By implementing the recently developed computational approach to EBID, the growth of complex 3D structures can be simulated and optimized prior to the actual deposition. ${ }^{29}$ Combining such simulations with the EBID Josephson junctions presented here provides an exciting opportunity for realizing 3D-printed superconducting circuits and quantum networks.

\section{METHODS}

Procedure for Printing a Josephson Junction. All junction devices are written using a $10 \mathrm{keV}$ electron beam with $20 \mathrm{nA}$ beam current, corresponding to a beam-spot diameter of around $60 \mathrm{~nm}$ in our instrument (Apreo SEM by Thermo Scientific with a gas injection system). The typical base pressure of the microscope chamber is about $2 \times 10^{-6}$ mbar. Once the $\mathrm{W}(\mathrm{CO})_{6}$ precursor is injected, the pressure increases to around $2 \times 10^{-5}$ mbar.

To print a Josephson junction, first, the superconducting electrodes are deposited onto a $\mathrm{Si}_{3} \mathrm{~N}_{4}$-coated silicon substrate by scanning the beam in a straight line (i.e., the "line scan mode"), applying $25 \mathrm{~ms}$ dwell time and $1 \mathrm{~nm}$ pitch (step size). The wires are written in a single row, separated by a gap of $160 \mathrm{~nm}$. Once the electrodes are formed, they are connected via a tungsten carbide weak link, deposited by raster scanning the beam over an area of approximately $0.5 \times 0.5 \mu \mathrm{m}^{2}$, using $23 \mathrm{~nm}$ step size. The dwell time used in writing the weak link is $4 \mathrm{~ms}$ for JJ1 and JJ2 and $10 \mathrm{~ms}$ for JJ3. To obtain transparent interfaces for efficient proximity, it is important to minimize the time interval between the formation of the electrodes and the deposition of the weak link.

Measurements. Transport measurements were performed in a variable-temperature cryostat, where sample temperature could be regulated between 300 and $1.5 \mathrm{~K}$. At low temperatures, the temperature stability of the system was better than $1 \mathrm{mK}$. Resistance and current-voltage $(I-V)$ characteristics were measured in a fourprobe configuration, using a current-biased circuit and a nanovoltmeter or, alternatively, a lock-in amplifier (both setups produced the same results). The Shapiro response of the junctions was obtained by measuring their $I-V$ characteristics while irradiating them with microwave frequencies from a nearby antenna. The procedure was 
repeated for multiple frequencies, including $f=6.4 \mathrm{GHz}$ (shown in the article). In each case, the height of the voltage steps varied in accordance to $f \Phi_{0}$. Magnetotransport measurements were taken by sweeping the field in the out-of-plane direction.

\section{ASSOCIATED CONTENT}

\section{s) Supporting Information}

The Supporting Information is available free of charge at https://pubs.acs.org/doi/10.1021/acsnano.0c03656.

Additional details on the superconducting transition and normalized resistivity versus temperature graph of wires deposited with $80 \mathrm{nA}$; transport measurements and SEM image of an inhomogeneous wire; Shapiro response of device JJ3; a table containing the parameters used in the fabrication of the $\mathrm{W}-\mathrm{C}$ wires and ribbons discussed in this work (PDF)

\section{AUTHOR INFORMATION}

\section{Corresponding Author}

Kaveh Lahabi - Kamerlingh Onnes Laboratory, Leiden University, 2300 RA Leiden, The Netherlands; 이잉.org/ 0000-0001-8070-7310; Email: lahabi@

physics.leidenuniv.nl

\section{Authors \\ Tycho J. Blom - Kamerlingh Onnes Laboratory, Leiden University, 2300 RA Leiden, The Netherlands \\ Thomas W. Mechielsen - Kamerlingh Onnes Laboratory, Leiden University, 2300 RA Leiden, The Netherlands \\ Remko Fermin - Kamerlingh Onnes Laboratory, Leiden University, 2300 RA Leiden, The Netherlands \\ Marcel B. S. Hesselberth - Kamerlingh Onnes Laboratory, Leiden University, 2300 RA Leiden, The Netherlands \\ Jan Aarts - Kamerlingh Onnes Laboratory, Leiden University, 2300 RA Leiden, The Netherlands; (1) orcid.org/0000- 0002-4113-0835}

Complete contact information is available at:

https://pubs.acs.org/10.1021/acsnano.0c03656

\section{Notes}

The authors declare no competing financial interest.

\section{ACKNOWLEDGMENTS}

This work is part of Project No. 680-91-128, which is (partly) financed by the Dutch Research Council (NWO).

\section{REFERENCES}

(1) Cybart, S. A.; Cho, E. Y.; Wong, T. J.; Wehlin, B. H.; Ma, M. K.; Huynh, C.; Dynes, R. C. Nano Josephson Superconducting Tunnel Junctions in $\mathrm{YBa}_{2} \mathrm{Cu}_{3} \mathrm{O}_{7-\delta}$ Directly Patterned with a Focused Helium Ion Beam. Nat. Nanotechnol. 2015, 10, 598-602.

(2) Gibson, J. W.; Hein, R. A. Superconductivity of Tungsten. Phys. Rev. Lett. 1964, 12, 688-690.

(3) Kondo, S. Supercondcucting Characteristics and the Thermal Stability of Tungsten-Based Amorphous Thin Films. J. Mater. Res. 1992, 7, 853-860.

(4) Sadki, E. S.; Ooi, S.; Hirata, K. Focused Ion Beam Induced Deposition of Superconducting Thin Films. Phys. C 2005, 426-431, $1547-1551$.

(5) Luxmoore, I.; Ross, I.; Cullis, A.; Fry, P.; Orr, J.; Buckle, P.; Jefferson, J. Low Temperature Electrical Characterisation of Tungsten Nano-Wires Fabricated by Electron and Ion Beam Induced Chemical Vapour Deposition. Thin Solid Films 2007, 515, 6791-6797.
(6) Li, W.; Fenton, J. C.; Wang, Y.; McComb, D. W.; Warburton, P. A. Tunability of the Superconductivity of Tungsten Films Grown by Focused-Ion-Beam Direct Writing. J. Appl. Phys. 2008, 104, No. 093913.

(7) Dai, J.; Onomitsu, K.; Kometani, R.; Krockenberger, Y.; Yamaguchi, H.; Ishihara, S.; Warisawa, S. Superconductivity in Tungsten-Carbide Nanowires Deposited from the Mixtures of $\mathrm{W}(\mathrm{CO})_{6}$ and $\mathrm{C}_{14} \mathrm{H}_{10}$. Jpn. J. Appl. Phys. 2013, 52, No. 075001.

(8) Ballestar, A.; Esquinazi, P. Transport Characteristics of Focused Beam Deposited Nanostructures. Nanofabrication 2015, 2, 1-18.

(9) Dai, J.; Xie, S.; Chang, H.; Guo, D.; Kometani, R. A SemiEmpirical Growth Model Study of W-C Induced by Focused Ion Beam with a Gaussian-Holtsmarkian Distribution. J. Mater. Sci. 2017, 52, 12326-12335.

(10) Córdoba, R.; Ibarra, A.; Mailly, D.; De Teresa, J. M. Vertical Growth of Superconducting Crystalline Hollow Nanowires by $\mathrm{He}+$ Focused Ion Beam Induced Deposition. Nano Lett. 2018, 18, 13791386.

(11) Córdoba, R.; Mailly, D.; Rezaev, R. O.; Smirnova, E. I.; Schmidt, O. G.; Fomin, V. M.; Zeitler, U.; Guillamón, I.; Suderow, H.; De Teresa, J. M. Three-Dimensional Superconducting Nanohelices Grown by $\mathrm{He}^{+}$-Focused-Ion-Beam Direct Writing. Nano Lett. 2019, 19, 8597-8604.

(12) Kohlmann-von Platen, K. T.; Buchmann, L. M.; Petzold, H. C.; Brünger, W. H. Electron-Beam Induced Tungsten Deposition: Growth Rate Enhancement and Applications in Microelectronics. J. Vac. Sci. Technol., B: Microelectron. Process. Phenom. 1992, 10, 26902694.

(13) Huth, M.; Klingenberger, D.; Grimm, C.; Porrati, F.; Sachser, R. Conductance Regimes of W-Based Granular Metals Prepared by Electron Beam Induced Deposition. New J. Phys. 2009, 11, No. 033032.

(14) Sengupta, S.; Li, C.; Baumier, C.; Kasumov, A.; Guéron, S.; Bouchiat, H.; Fortuna, F. Superconducting Nanowires by ElectronBeam-Induced Deposition. Appl. Phys. Lett. 2015, 106, 042601.

(15) Winhold, M.; Weirich, P. M.; Schwalb, C. H.; Huth, M. Superconductivity and Metallic Behavior in $\mathrm{Pb}_{\mathrm{x}} \mathrm{C}_{\mathrm{y}} \mathrm{O}_{\delta}$ Structures Prepared by Focused Electron Beam Induced Deposition. Appl. Phys. Lett. 2014, 105, 162603.

(16) Makise, K.; Mitsuishi, K.; Shimojo, M.; Shinozaki, B. Microstructural Analysis and Transport Properties of $\mathrm{MoO}$ and MoC Nanostructures Prepared by Focused Electron Beam-Induced Deposition. Sci. Rep. 2015, 4, 5740.

(17) Porrati, F.; Barth, S.; Sachser, R.; Dobrovolskiy, O. V.; Seybert, A.; Frangakis, A. S.; Huth, M. Crystalline Niobium Carbide Superconducting Nanowires Prepared by Focused Ion Beam Direct Writing. ACS Nano 2019, 13, 6287-6296.

(18) Hoyle, P. C.; Ogasawara, M.; Cleaver, J.; Ahmed, H. Electrical Resistance of Electron Beam Induced Deposits from Tungsten Hexacarbonyl. Appl. Phys. Lett. 1993, 62, 3043-3045.

(19) Van Dorp, W. F.; Hagen, C. W. A Critical Literature Review of Focused Electron Beam Induced Deposition. J. Appl. Phys. 2008, 104, 081301.

(20) Mongillo, M.; Jansen, L.; Audoit, G.; Berthier, R.; Cooper, D. Electronic Transport on W-Rich Films Deposited by Focused Ion Beam. J. Supercond. Novel Magn. 2017, 30, 2261-2270.

(21) Aloysius, R. P.; Husale, S.; Kumar, A.; Ahmad, F.; Gangwar, A. K.; Papanai, G. S.; Gupta, A. Superconducting Properties of Tungsten Nanowires Fabricated Using Focussed Ion Beam Technique. Nanotechnology 2019, 30, 405001.

(22) Guillamón, I.; Suderow, H.; Fernández-Pacheco, A.; Sesé, J.; Córdoba, R.; De Teresa, J. M.; Ibarra, M. R.; Vieira, S. Direct Observation of Melting in a Two-Dimensional Superconducting Vortex Lattice. Nat. Phys. 2009, 5, 651-655.

(23) Kes, P. H.; Tsuei, C. C. Two-Dimensional Collective Flux Pinning, Defects, and Structural Relaxation in Amorphous Superconducting Films. Phys. Rev. B: Condens. Matter Mater. Phys. 1983, 28, $5126-5139$. 
(24) Angers, L.; Chiodi, F.; Montambaux, G.; Ferrier, M.; Gueron, S.; Bouchiat, H.; Cuevas, J. C. Proximity DC Squids in the LongJunction Limit. Phys. Rev. B: Condens. Matter Mater. Phys. 2008, 77, 165408.

(25) Chiodi, F.; Ferrier, M.; Guéron, S.; Cuevas, J. C.; Montambaux, G.; Fortuna, F.; Kasumov, A.; Bouchiat, H. Geometry-Related Magnetic Interference Patterns in Long SNS Josephson Junctions. Phys. Rev. B: Condens. Matter Mater. Phys. 2012, 86, No. 064510.

(26) Dobrovolskiy, O. V.; Vodolazov, D. Y.; Porrati, F.; Sachser, R.; Bevz, V. M.; Mikhailov, M. Y.; Chumak, A. V.; Huth, M. Ultra-Fast Vortex Motion in a Direct-Write Nb-C Superconductor. Nat. Commun. 2020, 11, 3291.

(27) Fowlkes, J. D.; Winkler, R.; Lewis, B. B.; Stanford, M. G.; Plank, H.; Rack, P. D. Simulation-Guided 3D Nanomanufacturing via Focused Electron Beam Induced Deposition. ACS Nano 2016, 10, $6163-6172$.

(28) Keller, L.; Huth, M. Pattern Generation for Direct-Write ThreeDimensional Nanoscale Structures via Focused Electron Beam Induced Deposition. Beilstein J. Nanotechnol. 2018, 9, 2581-2598.

(29) Winkler, R.; Fowlkes, J. D.; Rack, P. D.; Plank, H. 3D Nanoprinting via Focused Electron Beams. J. Appl. Phys. 2019, 125, 210901. 